\title{
Osteopetrosis with micro-lacunar resorption because of defective integrin organization
}

\author{
Harry C Blair ${ }^{1,2}$, Beatrice B Yaroslavskiy², Lisa J Robinson², Markus Y Mapara ${ }^{3}$, Alessandra Pangrazio ${ }^{4,5}$, Lida Guo ${ }^{2}$, \\ Ka Chen ${ }^{2}$, Paolo Vezzoni ${ }^{4,5}$, Jakub Tolar ${ }^{6}$ and Paul J Orchard ${ }^{6}$
}

In vitro differentiated monocytes were used to characterize the cellular defect in a type of osteopetrosis with minimally functional osteoclasts, in which defects associated with common causes of osteopetrosis were excluded by gene sequencing. Monocytes from the blood of a 28-year-old patient were differentiated in media with RANKL and CSF-1. Cell fusion, acid compartments within cells, and tartrate resistant acid phosphatase (TRAP) activity were normal. However, the osteoclasts made abnormally small pits on the dentine. Phalloidin labeling showed that the cell attachments lacked the peripheral ring structure that supports lacunar resorption. Instead, the osteoclasts had clusters of podosomes near the center of cell attachments. Antibody to the $\alpha v \beta 3$ integrin pair or to the C-terminal of $\beta 3$ did not label podosomes, but antibody to $\alpha \mathrm{v}$ labeled them. Western blots using antibody to the $\mathrm{N}$-terminal of $\beta 3$ showed a protein of reduced size. Integrins $\beta 1$ and $\beta 5$ were upregulated, but, in contrast to observations in $\beta 3$ defects, $\alpha 2$ had not increased. The $\rho$-GTP exchange protein Vav3, a key attachment organizing protein, did not localize normally with peripheral attachment structures. Vav3 forms of $70 \mathrm{kD}$ and $90 \mathrm{kD}$ were identified on western blots. However, the proteins $\beta 3$ integrin, Vav3, Plekhm1, and Src, implicated in attachment defects, had normal exon sequences. In this new type of osteopetrosis, the integrin-organizing complex is dysfunctional, and at least two attachment proteins may be partially degraded.

Laboratory Investigation (2009) 89, 1007-1017; doi:10.1038/labinvest.2009.58; published online 22 June 2009

KEYWORDS: osteopetrosis; bone resorption; integrin assembly; receptor activator of NF- $\kappa$ B; $\rho$-GTPase

Genetic disorders that compromise bone degradation cause osteopetrosis. ${ }^{1}$ Without remodeling of mineralized cartilage and bone, long bones are packed with mineralized matrix. Mature lamellar bone cannot replace woven bone, causing a high frequency of fractures. Fractures heal poorly and often with deformity. In severe cases, the optic nerve is entrapped shortly after birth with consequent blindness. In all but the mildest forms, reduction of bone marrow causes anemia and hepatosplenomegaly due to extramedullary hematopoiesis. Osteopetrosis associated with hematological dysfunction is progressive and may need allogeneic hematopoietic stem cell transplant, which has the capacity to produce osteoclasts capable of remodeling the skeleton.

Osteopetrosis is rare but has been studied extensively. Most cases of human osteopetrosis have molecular diagnoses. The central activity of the osteoclast is acid transport to dissolve bone mineral, driven by a vacuolar-type $\mathrm{H}^{+}-\mathrm{ATPase}^{2}$ one subunit of which is an osteoclast-specific isoform. ${ }^{3}$ Mutations in the TCIRG1 gene coding for this isoform are the predominant cause of human osteopetrosis. ${ }^{4}$ The v-type $\mathrm{H}^{+}$ATPase is electrogenic, so anion transport must exist to balance $\mathrm{H}^{+}$for the acid secretion that dissolves bone mineral, and a chloride-proton exchanger, CLCN7, ${ }^{5}$ is also critical to osteoclast function. ${ }^{6}$ Defects in CLCN7 are responsible for many cases of osteopetrosis with normal TCIRG1, including dominant genotypes. CLCN7 needs a second membrane protein for its function, OSTM $1 .^{7}$ Another protein needed for the formation of the acid-secreting apparatus is Plekhm1, which by homology is probably a small GTPase of the Ras family. ${ }^{8}$ There are other rare causes of sclerotic diseases of the bone, including defects in acidproteinase activity and a number of defects in osteoclast differentiation, mostly known only from knockout mice. ${ }^{9}$ However, most cases of osteopetrosis have many nonfunc-

\footnotetext{
${ }^{1}$ Department of Pathology, University of Pittsburgh, and Veteran's Affairs Medical Center, Pittsburgh, PA, USA; ${ }^{2}$ Department of Cell Biology and Physiology, University of Pittsburgh, and Veteran's Affairs Medical Center, Pittsburgh, PA, USA; ${ }^{3}$ Department of Medicine, University of Pittsburgh, and Veteran's Affairs Medical Center, Pittsburgh, PA, USA; ${ }^{4}$ Istituto di Tecnologie Biomediche, CNR, Segrate, Italy; ${ }^{5}$ sttituto Clinico Humanitas, IRCCS, Rozzano, Italy and ${ }^{6}$ Division of Blood and Marrow Transplantation, Department of Pediatrics, University of Minnesota, Minneapolis, MN, USA

Correspondence: Professor HC Blair, MD, Department of Pathology, University of Pittsburgh, 705 Scaife Hall, Pittsburgh, PA 15261, USA.

E-mail: hcblair@imap.pitt.edu

Received 26 October 2008; revised 24 April 2009; accepted 26 April 2009
} 
tional osteoclasts, whereas in rare cases with few or no osteoclast defects in RANKL, a TNF-family protein that is a key osteoclast differentiation signal, have been identified. ${ }^{10}$ On the other hand, studies of organizing proteins of the $\rho$-GTP exchange family have shown that Vav3 is needed for normal osteoclast attachment, and that Vav3-knockout mice have an osteopetrotic phenotype without major developmental defects in other organ systems. ${ }^{11}$ One cause of osteoclastpoor osteopetrosis has recently been shown to be defects in RANK signalling. ${ }^{12}$

Despite these advances in the molecular etiology of osteopetrosis, about $20 \%$ of cases do not show any known defect. Some cases have defied systematic classification based on current developmental pathways; this work studies the basis of one such with normal RANK and acid transport, but an osteoclast attachment defect. Osteoclast attachment is mediated by the $\alpha \mathrm{v} \beta 3$ integrin, and the attachment is linked to complex additional proteins that create a tight annular bone adhesion zone at the periphery of the osteoclast. Without this attachment, the bone cannot be acidified and degraded. However, defects in attachment integrins that are not lethal during embryonic development are usually mild because of compensatory expression of integrins with similar function. ${ }^{13}$ Here we describe a form of osteopetrosis that does not fit this paradigm. Osteoclasts could be differentiated in vitro and resorb bone. However, the osteoclast podosome distribution was highly disordered, and only very small resorption lacunae were made by the cells. The affected osteoclasts make the $\alpha \mathrm{v}$ and $\beta 3$ integrins, but integrin assembly was abnormal, probably because of a defect in the organizing complex affecting its stability.

\section{MATERIALS AND METHODS}

\section{Osteopetrotic Cells and Genetic Sequencing}

Cells were from the peripheral blood of a 28 -year-old adult osteopetrotic patient; controls used cells from healthy blood donors. The patient was diagnosed at 3 years of age, and developed chronic osteomyelitis of the mandible and the left femur, and multiple fractures of long bones. Radiographs showed osteosclerosis, and osteopetrosis was confirmed by biopsy. Osteoclasts were present, and common causes of osteopetrosis were excluded by normal CLCN7 and TCIRG1 sequences from PCR-amplified cDNA, ${ }^{14}$ carried out as clinical tests (Connective Tissue Gene Tests, Allentown, PA, USA). The patient had normal hemostasis into young adulthood and despite chronic infectious complications, was stable with conservative management. The decision to transplant at the age of 28 was because of progressive splenomegaly, leukoerythroblastosis, and thrombocytopenia. Before transplant, $40 \mathrm{ml}$ of blood was taken for the analysis of osteoclastic function by in vitro osteoclast differentiation in a protocol approved by the Institutional Review Board. Further sequencing of gene defects that are reported to cause defects related to the in vitro findings was done using PCR-amplified DNA with the primer pairs specified in Table 1.

\section{Cell Culture}

In vitro osteoclast generation used $40 \mathrm{ml}$ of heparin anticoagulated blood. Control osteoclasts were made from citrate anti-coagulated buffy coats saved from leukoreduction of the donor blood. Mononuclear cells were recovered on ficoll, and CD14 osteoclast precursors were enriched using anti-CD14 magnetic microbeads (Miltenyi Biotec, Auburn CA, USA) as described. ${ }^{4}$ To induce osteoclast differentiation, media were supplemented with $10 \mathrm{ng} / \mathrm{ml} \mathrm{CSF-1}$ and $40 \mathrm{ng} / \mathrm{ml}$ recombinant RANKL, and media were changed twice weekly. Cells were grown on glass coverslips in $0.5 \mathrm{~cm}^{2}$ wells. For resorption assays, $5 \mathrm{~mm}$ diameter whale-dentine discs, $1 \mathrm{~mm}$ thick, were added to the tissue culture wells. Mononuclear cells recovered on ficoll are approximately $50 \%$ CD14 or CD11 monocytes, whereas CD14 affinity purified cells are approximately $98 \%$ monocytes by flow analysis, as shown previously. ${ }^{15}$ Other cells in ficoll-separated buffy coats include lymphocytes and some polymorphonuclear leukocytes, which do not attach to glass and do not represent significant numbers of cells in fully differentiated osteoclast cultures. Typically, at the time of study $50 \%$ of cells in cultures are mononuclear, and the remainder are multinucleated osteoclasts, regardless of whether ficoll buffy coat cells or CD14 purified monocytes are used for production. Only the multinucleated, tartrate resistant acid phosphatase (TRAP) expressing cells were considered in studies of cell adhesion.

\section{Fluorescent Antibody Labeling and Western Analysis}

Anti-vitronectin receptor CD51/CD61 $(\alpha \mathrm{v} \beta 3)$ mouse monoclonal anti-human 23C6 sc-7312, $\alpha \mathrm{v}$ rabbit polyclonal antibody sc-10719, $\beta 1$ (N-terminal anti-20mer, sc-6622) goat anti- $\beta 3$ (N-terminal anti $20 \mathrm{mer}$ sc-6627) goat polyclonal antibody, $\beta 3$ C-terminal mouse monoclonal antibody 611140, $\beta 5$ (E19 anti N-terminal peptide, sc-5401) and $\alpha 2$ (N-terminal anti 19mer, sc-6585) were anti-human antibodies. Antibodies to integrins and to the vitronectin receptor were from Santa Cruz (Santa Cruz, CA, USA) except for the $\beta 3 \mathrm{C}$-terminal antibody, which was from BD Transduction laboratories (San Jose, CA, USA). Antibody to Vav3 was a goat polyclonal (ab21208) from AbCam (Cambridge, MA, USA) prepared to the synthetic human peptide CSGEQGTLKLPEK and was used at $1 \mu \mathrm{g} / \mathrm{ml}$ for western blot and $3 \mu \mathrm{g} / \mathrm{ml}$ for immune localization. Other antibodies were used for immune fluorescence at 1:100 dilution and for western blotting at 1:200. Al-488-conjugated goat anti-mouse or donkey anti-goat second antibodies were from Jackson Immunoresearch (Westgrove, PA, USA) and rhodamineconjugated phalloidin for labeling filamentous actin was from Molecular Probes (Eugene, OR, USA). Immune fluorescence labeling was carried out as described. ${ }^{12}$ For western blots cell lysates were made using $0.3 \%$ SDS, $50 \mathrm{mM}$ tris, $\mathrm{pH} 7$, with proteinase, and phosphatase inhibitors. Proteins were separated on 8 or 10\% SDS-PAGE and transferred to polyvinylidine membranes. Primary antibodies were, for detection on western blots, used with alkaline phosphatase- 
Table 1 Primers for sequencing

\begin{tabular}{|c|c|c|}
\hline $\begin{array}{l}\text { Gene symbol } \\
\text { Exon/ }\end{array}$ & Forward primer & Reverse primer \\
\hline \multicolumn{3}{|l|}{$S R C$} \\
\hline 1 & GGGCCTCGTITTCCCTATCT & CCAGGCAACGAAAAAGGACT \\
\hline 2 & CACGGATGCAGTGGACACGT & GATACTTGGGTGGGCTGACG \\
\hline 3 to 4 & AGATGACCAAGGCCAGTCCT & ATCCTGCCATTACCTCCACC \\
\hline 5 & ССТСССТTСССТССAATGTC & GTCCCCACCCTTCTAGGCTC \\
\hline 6 & TGCGTGTCCCCTGAAATCTG & GTCACCAGCCCCATTCACAG \\
\hline 7 & TAACAGTGCCATGAGGAGCA & CTACTTGGGTGGCTGAGGTG \\
\hline 8 & TCTGCTGTGCTGGTTAAAGC & GTAAATACGTGCGGCAAGAT \\
\hline 9 to 10 & GAAGAAGTGTGGGGAGGGTG & GCCTAACATCAGACGCTCAA \\
\hline 11 & TTGAGCGTCTGATGTTAGGC & CCCACAGAGAGAGGACCAAC \\
\hline \multicolumn{3}{|l|}{ VAV3 } \\
\hline 1 & GGGACCCCATCGTATTACAG & CGCATGATTAATGGGAGCTG \\
\hline 2 & AAAGGCTGCTTGATGCTTTC & TGGTITGCTAAGCCATCTACA \\
\hline 3 & GCCCTTCTCATTCTGCACAT & AAGAAGAACCACACCAGTGACA \\
\hline 4 & GAGCAGAAATGTCAGGGTCA & AAAACCCACAATGGACACAT \\
\hline 5 & CACTGCCCTCGTTATTGGAT & GGTCATGCATATGTCTCCAAA \\
\hline 6 & CAGCAAAGATGCCTCACAAA & CTGAAACAGTGCCACTCAGG \\
\hline 7 & TCCTTCCTITTGGAAGCATT & GGAACCCCCTAAGGAAATGA \\
\hline 8 & CCTGAGTGGCAAGTCTAGCA & TGAAAGTAATTGTCCCACGTTC \\
\hline 9 & GTTTCCCCCTCCAGTTCATT & CATGTAAAACACAGGGCCATAA \\
\hline 10 & CCTAGGGCCTITGCATCTTा & GGCAGCTACTGACCATCTCTG \\
\hline 11 & GGAAATGGAGCCCAGACATA & CCATGCAAGGATAACTATTCCA \\
\hline 12 & GCTITGATTGACTGGGCATT & GGGACAAGCCATCACAAAGT \\
\hline 13 & ACCCTTCCGGTTGAGTCTTI & TICCCCCTACTTCACACTGG \\
\hline 14 to 15 & CCCCGTTGTTTCAGTGTATG & TCCCGTCGCTGTACACATTA \\
\hline 16 to 17 & TTGCAGCTITGTTGTGAAAC & СCATCCAACTCCCTCTTGAA \\
\hline 18 & GCATGTTGAAAGAAAGGTTGC & CCTTCGGACAGTGACAGTGA \\
\hline 19 & TGCTITCCAAAGTGCCAGTA & AGGGTGCTGGATATGTGAGC \\
\hline 20 & TGTCAGGGATCATGGGATAAA & TTGCTITGCACTGTGGACTC \\
\hline 21 & TTGCTTCCTITTCTGATCCAC & TGACCTGGCCAGTATCCTIT \\
\hline 22 & GTTCCAGGACTGTGCCACTT & AGCCGAGACAAATGGAAATG \\
\hline 23 to 24 & GCCTCTGTTCTCTTCCAGGT & TGAAGATTACGATTCGAGCA \\
\hline 25 & TCCAGACAGCAGGGAGAGAT & GCCTGTTGGTCCATCTGACT \\
\hline 26 to 27 & CGAACTGCAAACCTACTGTCA & TGTTGAACAGCCAGAAATGC \\
\hline PLEKHM1 & As reported ${ }^{8}$ & \\
\hline \multicolumn{3}{|l|}{ ITGB3 } \\
\hline 1 & GGCGAGAGAGGAGCAAAGTT & GCTCCA AGTCCGCAACTTGAC \\
\hline 2 & GCAAGTACCTTCACTGAGCCA & AGGGAGGTGATAACTTCATGG \\
\hline 3 & GGTAGAGAGTCGCCATAGTTC & CAAGACTTCCTCCTCAGACCT \\
\hline 4 & CCCAACTGTATCCAAATCTGC & AACCAGGACTTGGACCTTCCT \\
\hline 5 & ТССССТСТCTाTCCTTCCCAA & TTCAGTCACTTCCTITCCCAG \\
\hline 6 & TCTCTACCAGTGACATGGCTG & СTCTGCCTAGATTAGGGCAAC \\
\hline 7 & GCAGCCCGAAGCAAGATAAGT & TGGGGACTGTATCCAATGCTA \\
\hline 8 & GCTाTGGAGACCACCAGCTT & AGCCCTGCTGTGTAAGCCAAA \\
\hline 9 & TGCTCTTGCATTTCCCGTTC & GGGGCCATIITAGTCACTAC \\
\hline 10 & GGCCCAACTGTGTCTAAATAC & TGCAGGTATATGAGGGGGTGT \\
\hline 11 to 12 & TGTGTAGCCTGCTGCCATGGA & ACCTGGGTGTGTGCAACTCTA \\
\hline 12 & GATCAGAGCTGGACTGGGATA & ACCTGGGTGTGTGCAACTCTA \\
\hline 13 & GTTGGAGTGGTCCCATCTTC & GAGATTCCTGCCTAACATGGT \\
\hline 14 & GGACCTTAGTTACTITGTC & ATCTCTCCCAGTTTCCAGA \\
\hline 15 & AGAGAACGGTGCCTTGGGAAA & ACACACTCCCACACACAACAC \\
\hline
\end{tabular}

Primers were designed in the flanking introns (or in flanking regions for the first and last primers). Sequences are from GenBank; the header of each section is the GenBank gene name; the sequences are from that gene file for Homo sapiens. coupled secondary antibodies (Pierce immuno-pure polyclonal antibodies, Thermo-Fisher Rockford IL, USA) followed by enhanced chemiluminescence detection (ECL plus, Amersham, Piscataway, NJ, USA) as described. ${ }^{16}$ Efficency of osteoclast formation in vitro between control and osteopetrotic precursor cells, using similar densities of attached monocytes appeared to be the same within a factor of two. The fusion efficiency could not be determined more accurately because of the limited number of osteopetrotic precursor cells available.

\section{Enzyme Activity, Acid Transport, and Pit Assays}

Tartrate resistant acid phosphatase was assayed in cells fixed in citrate buffer at pH 5.4 with $40 \%$ acetone. Acid phosphatase activity was then determined using naphthol AS-BI phosphate substrate with fast garnet GBC to visualize the product as a red precipitate in $0.67 \mathrm{M}$ tartrate at $\mathrm{pH} 5.6$ (Sigma, St Louis, MO, USA). Acid vesicles in osteoclasts were identified using the fluorescent polyamine-pyrrole dye lysotracker red DND-99 (Molecular Probes, Eugene, OR, USA), $2.5 \mu \mathrm{M}$, added $15 \mathrm{~min}$ before observation. The dye diffuses through membranes at neutral $\mathrm{pH}$. Its amines are protonated at low $\mathrm{pH}$ and accumulate in acid compartments, which are not permeable to ions. Lacunar resorption was assessed after culturing osteoclasts on slices of whale dentine for 12 days. ${ }^{17}$ The substrate was cleaned by ultrasonic disruption in $1 \%$ sodium borate for $5 \mathrm{~min}$ to remove cell debris. The substrate was then stained for $2 \mathrm{~min}$ with $1 \%$ toluidine blue to label the surface collagen exposed by degradation.

\section{Microscopy}

Images were obtained on a Nikon TE2000 inverted phasefluorescence microscope using a 12-bit $1600 \times 1200$ pixel monochrome charge coupled device with an RGB filter wheel for color photographs (Spot Instruments, Sterling Heights, MI, USA). For green fluorescence, excitation was at 450$490 \mathrm{~nm}$ with a $510 \mathrm{~nm}$ dichroic mirror and a $520 \mathrm{~nm}$ barrier. For red fluorescence (including lysotracker red), excitation was at $536-556 \mathrm{~nm}$ with a $580 \mathrm{~nm}$ dichroic mirror and a $590 \mathrm{~nm}$ barrier. For blue fluorescence, excitation was at 380$425 \mathrm{~nm}$ with a $430 \mathrm{~nm}$ dichroic filter and a $450 \mathrm{~nm}$ barrier. Fluorescent labels were photographed using $1.3 \mathrm{NA} \times 40$ or $\times 100$ oil objectives.

\section{RESULTS}

\section{Features of the Osteopetrotic Bone}

The patient was 28 -years old at the time of the study. PCRbased sequencing for defects in TCIRG1 or CLCN7, the common clinical causes of osteopetrosis, was negative. The iliac crest had a markedly abnormal medullary cavity with matrix consisting of calcified cartilage and woven bone (Figure 1a). Although the patient had severe hematological abnormalities, including leukoerythroblastosis and thrombocytopenia, the complication of blindness had been avoided. This may be due to residual surface degradative activity. 
This is supported by the observation at high power of osteoclasts with scalloped lacunae (Figure $1 \mathrm{~b}$ ) that were much smaller than those of normal osteoclasts, which cut the lacunae roughly to the size of the diameter of the cell.

\section{Osteoclasts Differentiated In Vitro}

Osteoclast CD14 precursors were isolated from blood of the patient and plated on whale dentine or glass, in parallel with control cells from normal blood donors. The osteoclast derived from osteopetrotic mononuclear cells made clear, but very small, pits (Figure $2 \mathrm{a}$ ) that had on average $\sim 10 \%$ of the area of pits from normal cells (Figure $2 \mathrm{~b}$ ). The micro-pits from the osteopetrotic cells resembled micro-lacunar resorption produced by human monocytes in TNF- $\alpha$ rather than RANKL, ${ }^{18}$ but the cells were as extensively multinucleated and spread as normal osteoclasts (Figure 2c), and produced the typical osteoclast enzyme TRAP in large quantities (Figure $2 \mathrm{~d}$ ). In keeping with the normal sequence of major acid-transport proteins, vacuolar acidification by lysotracker labeling of acid compartments appeared typical for osteoclasts in vitro (Figure 2e).

\section{Structural Anomalies in Membranes and Attachment Structures}

At high magnification, osteopetrotic osteoclasts had prominent membrane structures in central regions of the cells (Figure 3a), which did not occur in control osteoclasts. It could not be determined with certainty whether these addi- tional membrane images in phase were within or on the border of the affected cells. Similar structures were seen in phalloidin labeling as large concentrations of actin-labeled membranes (Figure 3c), which also showed large clusters and bands of podosomes, in many cases across the center of the osteoclastic cell attachments. In contrast, normal osteoclasts (Figure $3 \mathrm{~b}$ and $\mathrm{d}$ ) had well-defined peripheral rings of podosomes, no increased membranes in the cell center on phase, and no irregular bands of phalloidin labeling.

\section{Integrins in Osteopetrotic and Control Osteoclasts}

Osteopetrotic cells were labeled for actin with phalloidin (red) and antibodies for the $\alpha \mathrm{v} \beta 3$ integrin pair or $\alpha \mathrm{v}$ and $\beta 3$ subunits (green), and with Hoechst dye to visualize nuclei (Figure 4a). The affected cells had prominent podosomes, but the distribution of podosomes was abnormal. There were many podosomes in the cell centers, whereas in normal cells the podosomes are organized in annular rows associated with actin rings. A monoclonal antibody for the $\alpha \mathrm{v} \beta 3$ integrin showed no colocalization with podosomes in osteopetrotic cells. Polyclonal antibodies to $\alpha \mathrm{v}$ showed colocalization with podosomes (Figure $4 \mathrm{a}$ ) but $\beta 3$ integrin did not colocalize using antibody to the $\mathrm{N}$-terminus. This and the lack of labeling with the monoclonal antibody recognizing the $\alpha \mathrm{v} \beta 3$ integrin pair, probably reflect abnormal processing of the $\beta 3$ subunit. That the podosomes in osteopetrotic cells expressed integrins in small central clusters suggested that the integrins are synthesized, but are not trafficked properly or are
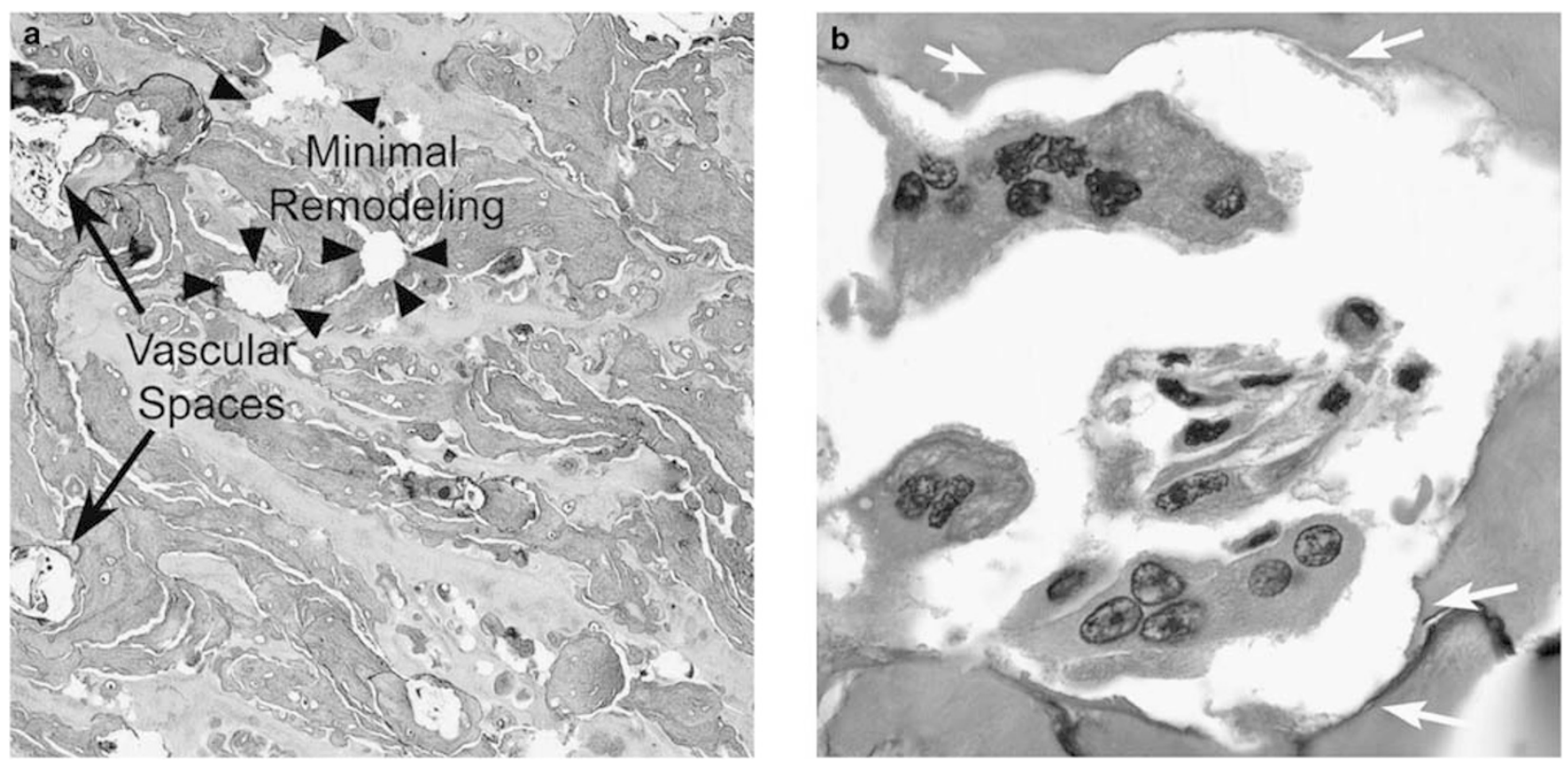

Figure 1 Features of the bone from a biopsy of the iliac crest. (a) Severe osteopetrosis with minimal remodeling (arrowheads) including some vascular spaces. Although the patient survived for 28 years before transplant, the major part of the bone was retained cartilage (lightly stained acellular material) with the balance mainly composed of primary spongiosa, and a few spaces. Low power ( $\times 10$ objective); the field shown is $800 \mu \mathrm{m}$ across. (b) Osteoclasts with shallow resorptive pits. Osteoclasts were present and appeared normal by light microscopy, although pits (arrows) were small and shallow relative to the number of multinucleated cells present. High power ( $\times 40$ objective); the field shown is $110 \mu \mathrm{m}$ across. 

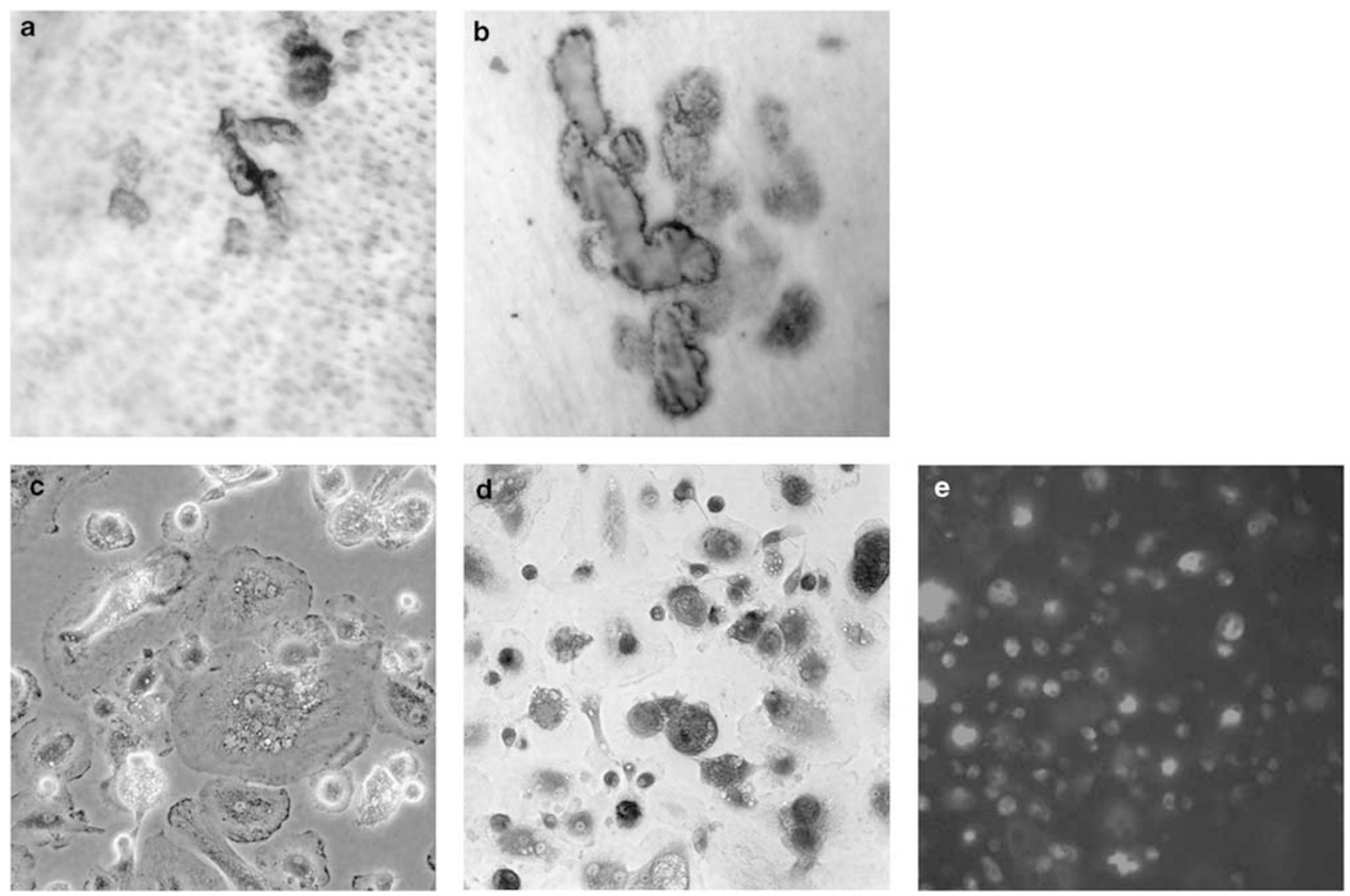

Figure 2 Osteoclasts formed in vitro from the osteopetrotic monocytes make small pits, but have normal multinucleation and tartrate resistant acid phosphatase (TRAP) expression. (a, b) Pit assays from osteopetrotic and control osteoclasts. Osteoclasts from the osteopetrotic patient produced pits (a), but the pits averaged $10-15 \mu \mathrm{m}$ in diameter, much smaller than the osteoclasts themselves (Figure 1 and $\mathbf{c}$, d below). Control osteoclasts produced pits averaging $40 \mu \mathrm{m}$ in diameter (b). Fields $450 \mu \mathrm{m}$ across ( $\times 20$ objective) are shown. Cells produced in vitro were incubated on whale dentine for 12 days. The substrate was cleaned by sonication and labeled with toluidine blue to show partially degraded matrix proteins at pit sites. (c) Osteopetrotic osteoclasts in vitro. Phase microscopy showed large multinucleated cells, typical of RANKL stimulated cell differentiation in vitro. The field shown is $280 \mu \mathrm{m}$ across. (d) tartrate resistant acid phosphatase (TRAP) activity of osteopetrotic osteoclasts formed in vitro. TRAP activity was completely typical of osteoclasts in vitro. This frames a bright-field image $450 \mu \mathrm{m}$ across, the same magnification as $\mathbf{a}$ and $\mathbf{b}$. Note that the pits in $\mathbf{b}$ have similar sizes to the large rounded cells expressing large amounts of TRAP, but that the pits in a are much smaller (see also Figure 1b). (e) Lysotracker red DND labeling. Cells on glass contain abundant acidic vacuoles, the same as in normal osteoclasts (not illustrated). ${ }^{4}$ The field is $450 \mu \mathrm{m}$ across.

unstable. In contrast, in control cells all of the integrin antibodies showed excellent podosome colocalization with phalloidin labeling (Figure $4 \mathrm{~b}$ ). Labeling for $\alpha 2$ did not label podosomes in control or osteopetrotic cells (not illustrated).

\section{Organizing Complexes for Integrin Assembly}

These findings indicated changes in integrin distribution. The changes in osteoclast function would be consistent with interference with organizing complexes. These are driven by actin and by $\rho$-type GTP exchange factors that regulate adhesion structure assembly and stability. The family of $\rho$-GTP exchange factor regulatory proteins include the Vav (Vav1) oncogene and BRCA1; the Vav3 $\rho$-GTP exchange factor was recently discovered to be critical to osteoclast attachment organization. ${ }^{11}$ By immune labeling, abundance and distribution of Vav3 labeling in control and osteopetrotic osteoclasts was studied (Figure 5). Surprisingly, Vav3 appeared to be, at least, as abundant in osteopetrotic and control cells, but unlike the normal Vav3 distribution, which occurs in punctate complexes surrounding podosomes and at actin organizing centers (top right panel), Vav3 in the osteopetrotic osteoclasts lacked specific association with these structures (bottom panels). This suggested an abnormal assembly of the Vav3 complex.

\section{Western Blots for Integrin Subunits and Vav3 in the Osteopetrotic Osteoclasts}

Size and quantity of $\alpha \mathrm{v}$ in the osteopetrotic cells matched that of normal osteoclasts (Figure 6). An N-terminal $\beta 3$ antibody detected only small amounts of $\beta 3$ in the osteopetrotic cells (Figure 6(1), whereas a C-terminal antibody showed normal quantities of $\beta 3$ of slightly smaller size (Figure 5(2), suggesting that the protein is abnormally processed, or that there is an N-terminal deletion. Possibilities included abnormal 

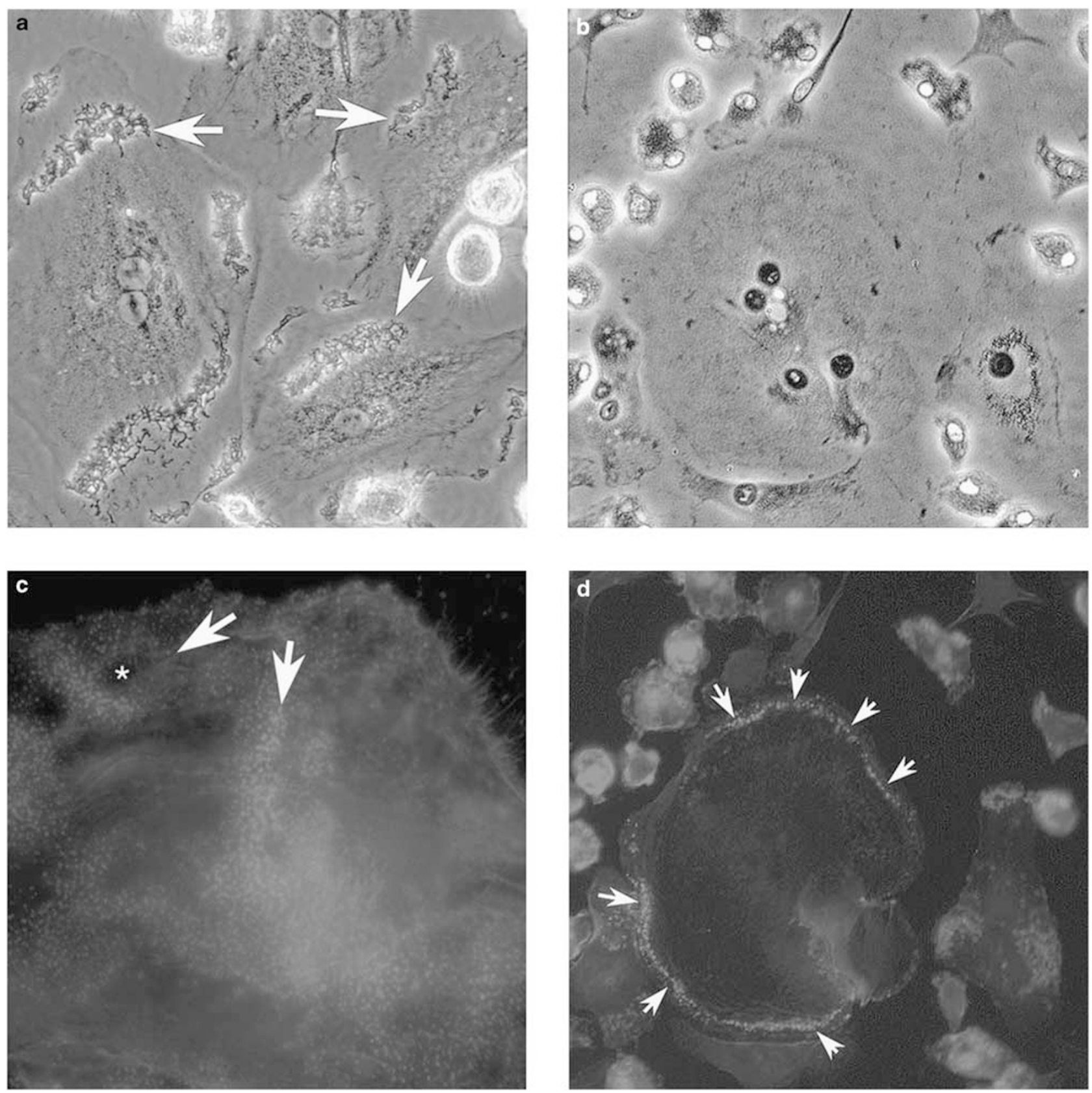

Figure 3 High-resolution phase and phalloidin rhodamine labeling show podosomal anomalies in osteopetrotic osteoclasts. Cells were grown on glass cover slips. The fields shown were taken with $\times 40$ objectives and all photographs are $220 \mu \mathrm{m}$ across. (a, c) Osteoclasts from the osteopetrotic patient. In phase contrast at high power (a) prominent cell membrane structures often occurred in broad regions of the cells (arrows). Phalloidin rhodamine labeling (c) showed very large clusters and bands of podosomes sometimes in peripheral bands and in some cases across the center of the cell attachment (arrows). These ringed some spaces (asterisk) but typically these were much smaller than the cell diameters, and highly variable. a and $\mathbf{c}$ are photographs of two similar fields. b, d control normal osteoclasts showing peripheral cell attachment structure. In contrast to osteopetrotic cells, normal osteoclasts have well defined borders in phase (b) that correspond to one or more regular and well-defined peripheral rings of podosomes (Arrowheads, $\mathbf{d}$ ). The cells shown in $\mathbf{b}$ and $\mathbf{d}$ are the same field.

$\alpha \mathrm{v} \beta 3$ assembly or stability, or a mutation (see below). The cell lysates were studied for alternative $\alpha$ and $\beta$ chains; $\beta 1$ was greatly increased and $\beta 5$ was somewhat increased in osteopetrotic cells (Figure 6(3). HeLa cells, where $\beta 1$ is a major adhesion protein, are shown as control. MG63 is shown as a control for $\beta 5$ expression. These findings suggested that the osteopetrotic cells responded to some extent by altering integrin synthesis. This also occurs in $\beta 3$ deletion (Glantzmann's thrombasthenia). ${ }^{13}$ However, the abnormal podosome distribution caused a much more serious 
a

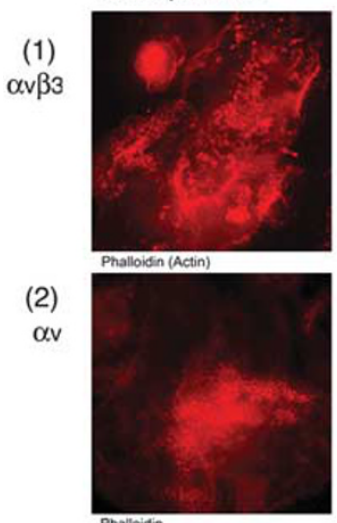

(3)

B3

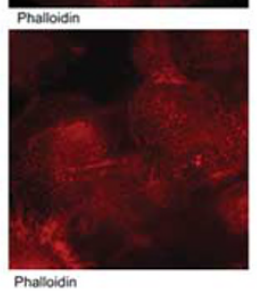

Osteoclasts
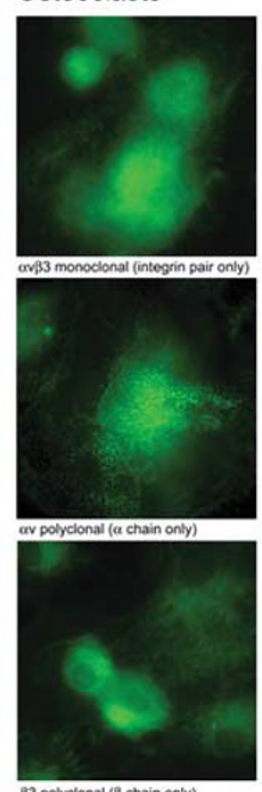
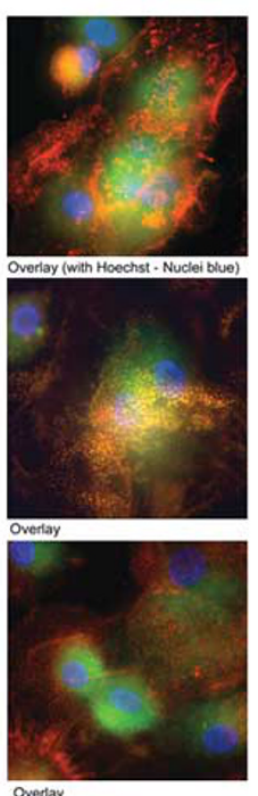

b Control Osteoclasts
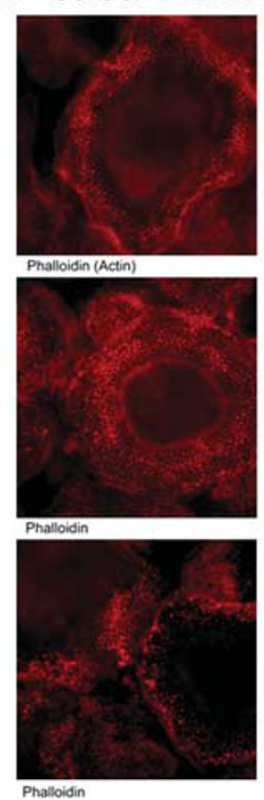
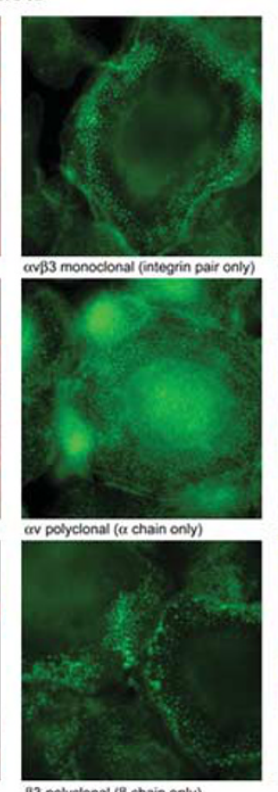

B3 polyclonal ( 3 chain only)
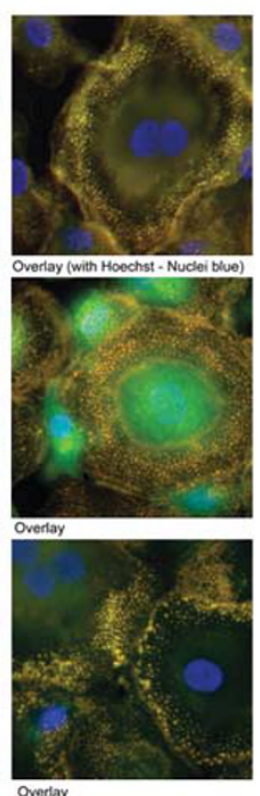

Figure 4 Integrin distribution in control and osteopetrotic osteoclasts. Cells grown as in Figures 1 and 2 were labeled for actin (phalloidin, red), antibodies to integrins specified (green), and Hoechst dye (nuclei, blue). Fields are each $85 \mu \mathrm{m}$ across. (a) Podosomes from osteoclasts of the affected patient label poorly for $\alpha \mathrm{v} \beta 3$ integrin or $\beta 3$ subunits, but include $\alpha \mathrm{v}$ integrin. The left column (red) shows actin with prominent podosomes in abnormal distribution (larger fields shown in Figure 3). Monoclonal antibody for the $\alpha \mathrm{v} \beta 3$ pair (1, top row) and polyclonal antibodies to $\alpha \mathrm{v}$ (2, middle row) and $\beta 3 \mathrm{~N}$-terminal ( 3 , bottom row) were used to label integrins (second column, green). There was essentially no colocalization $\alpha \mathrm{v} \beta 3$ with podosomes (third column, yellow shows colocalization). Labeling with anti-N-terminal $\beta 3$ was weak and did not colocalize with actin. This may reflect degradation of the $\beta 3 \mathrm{C}$-terminal (see Figure 6). There was colocalization of $\alpha \mathrm{v}$ with podosomes (third column from left), although podosomes were abnormally distributed as in Figure 3 . (b) Podosomes from normal osteoclasts label for $\alpha \mathrm{v} \beta 3$ integrin, and for $\alpha \mathrm{v}$ or $\beta 3$ integrin subunits. The photographs parallel those of the osteopetrotic cells (a). The osteoclasts have prominent peripheral rings of podosomes that label well with the integrin pair and with each integrin subunit (yellow in merged images, right column). No labeling of control or osteopetrotic cells for $\alpha 2$ integrin was detected in similar assays (not shown; $\alpha 2$ integrin is not expressed in major amounts in these cells, see Figure 6).

phenotype than results from $\beta 3$ deletion. In contrast, western blot for Vav $3^{11}$ showed a minor normal product of $\sim 90 \mathrm{kD}$ and a second band, only in osteopetrotic cells, at $\sim 70 \mathrm{kD}$. At this point no new lysate was available; Figures 6(4) and (5) are re-blots; the Vav3 blot was done in triplicate with identical results (two are shown). Here also, HeLa cells are shown as a control for normal Vav3. In $\beta 3$ defects $\alpha 2$ is also upregulated; ${ }^{13}$ in this case, $\alpha 2$ was a minor product and was not clearly increased. The $\alpha 2$ blot Figure 6(5) needed long exposures relative to other integrins, so it is likely that $\alpha 2$ was a small fraction of $\alpha$ integrin quantity in normal or osteopetrotic cells.

\section{Sequences of Genes Reported to Cause Abnormal Integrin Assembly}

Sequences of all exons of $\beta 3$ from the patient's genome matched the normal protein. In addition, all exons of Vav3, Src, and Plekhm1, which cause defects in osteoclast attachment organization in animal models (see Discussion) were sequenced. These also matched those of the normal human proteins. For sequencing primers and gene names see Table 1.

\section{DISCUSSION}

We characterized osteoclasts from a patient with severe osteopetrosis, surviving to adulthood but requiring transplant for hematopoietic system failure, with abnormal osteoclast attachment. Many attachment proteins are produced by cells of monocyte lineage during differentiation, and, although a number of abnormalities have been described in these attachment proteins, there are no human precedents for the functional defect described here. Most human osteopetrosis is due to defects in the specialized acid-secretion apparatus, probably because this relatively recent and specialized function has little overlap with other functions of acid secretion. ${ }^{9}$ There are many causes of osteopetrosis related to defective osteoclast maturation, but most are known only from transgenic animals. Clinically these will clearly be very rare, although two forms, related to either RANKL or RANK defect, do occur, and these cause osteoclast-poor osteopetrosis. $^{10,12,19}$

Human mononuclear osteoclast precursors occur in the monocyte fraction of the marrow and in peripheral blood. They express the integrins CD11b-c and the lipopolysaccharide receptor antigen $\mathrm{CD} 14,{ }^{20}$ but do not express the membrane-attachment proteins that characterize mature 

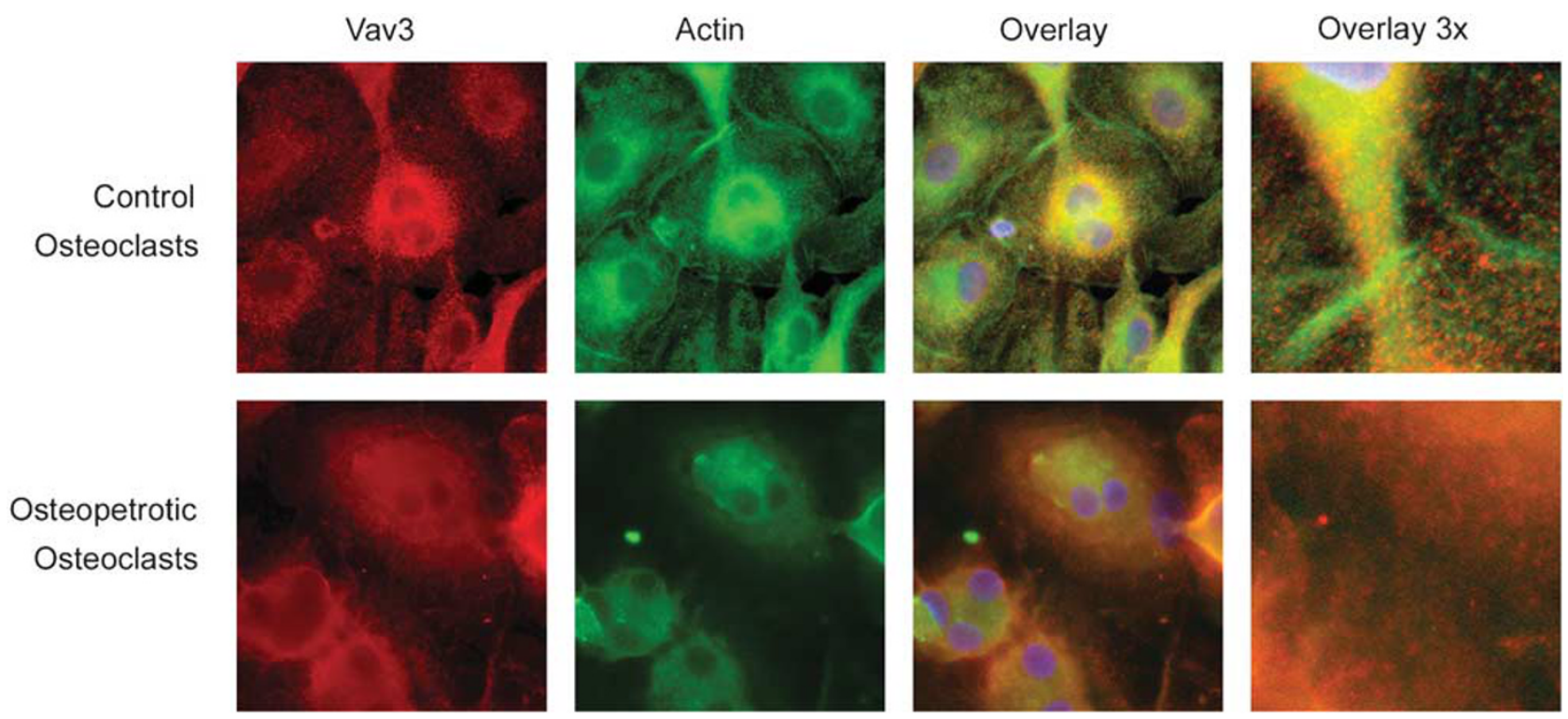

Figure 5 Dysfunctional distribution of Vav3 in osteopetrotic cells with abnormal attachment. Vav3 is critical to the organization of the cellular attachment and membrane receptors. Vav3 is associated with attachment structures in the periphery of control cells (top row). Vav3 was not associated with actin or podosomes in the periphery of osteopetrotic cells (bottom row). The relationship of Vav3, around the periphery of podosomes and near actin filaments, is seen most clearly at very high magnification power (right frames, $30 \mu \mathrm{m}$ across). Other frames are $85 \mu \mathrm{m}$ square.

(1)

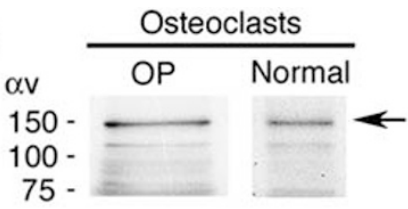

(2)

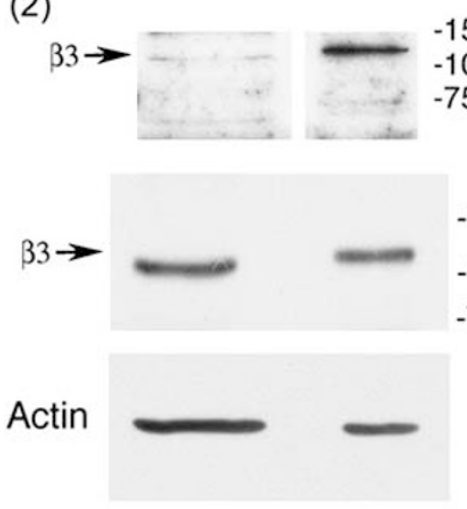

(3)

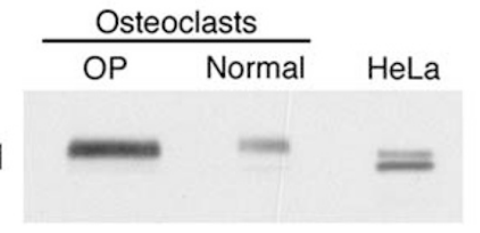

$-150$

$-100$

Actin

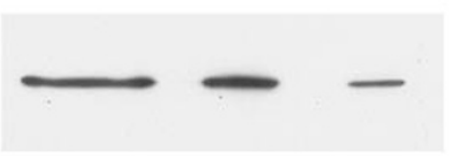

Osteoclasts

$-150$

$-100$

$-75$
OP Norma

$\beta 5$

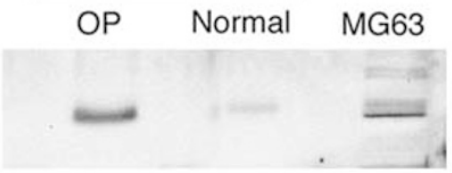

Actin
(4)

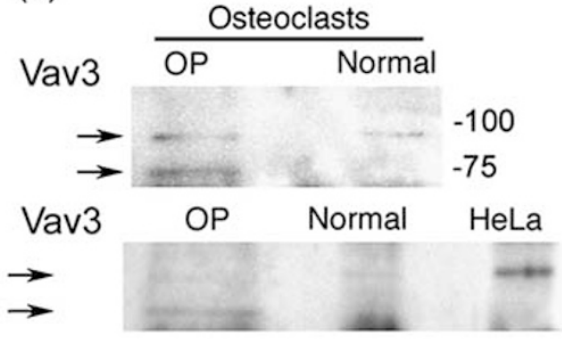

(5)

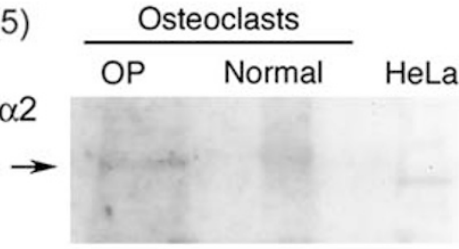

Figure 6 Western blots for integrin subunits and Vav3 in osteopetrotic osteoclasts and control cells. Blots used $10 \mu \mathrm{g}$ of cell-lysate protein separated on 8 or $10 \%$ SDS-PAGE and polyclonal antibodies visualized by enhanced chemiluminescence (see Methods). (1) Control and osteopetrotic cells showed similar results with antibody to the $\alpha \mathrm{v}$ integrin subunit. (2) Re-blot of the membrane (1) for the C-terminal region of the $\beta 3$ subunit was very weak in the osteopetrotic patient. A second blot using an antibody to the $\beta 3$ subunit $\mathrm{N}$-terminal region was showed similar $\beta 3$ in control and osteopetrotic cell (OP) cell lysates, although the $\beta 3$ is probably differently processed, as its nucleotide sequence was normal (see text). Actin re-blot is shown as a protein loading control in the second blot. Under the conditions used, actin is near the front and OP lysates, which contained more salt than controls, expanded toward the front. (3) Osteoclasts with $\beta 3$ defects have compensatory increases in $\beta 1$ or $\beta 5$. Additional blots using HeLa cells as a positive control for $\beta 1$ and MG63 cells as a control for $\beta 5$, as these integrin chains are expressed at low levels in normal osteoclasts. Both $\beta 1$ and $\beta 5$ were present in higher amounts in osteopetrotic cells than in normal controls. (4) Re-blots of membranes from (1) and (3) for Vav3. The OP patient showed two isoforms, one $\sim 20 \mathrm{kD}$ smaller than the normal form, which is also abundant in the HeLa cells. (5) The $\alpha 2$ integrin subunit is a minor protein, barely detectable, in normal or the OP osteoclasts. Re-blot of the membrane in (3) upper panel. This is in keeping with normal $\alpha \mathrm{v}$ expression, but contrasts with findings in patients with isolated $\beta 3$ defects (see text). Re-blot of (3). 
osteoclasts, principally the vitronectin receptor (the $\alpha \mathrm{v} \beta 3$ integrin). ${ }^{21,22}$ Absence of the $\alpha \mathrm{v}$ integrin chain, which is widely expressed and needed for normal vasculogenesis, is lethal in the embryonic period, ${ }^{23}$ whereas absence of the $\beta 3$ chain causes Glantzmann's thrombasthenia, a defect in platelet adhesion usually with minimal effects on bone degradation. Curiously, the principal substitute integrin pair is $\alpha 2 \beta 1$ in the absence of $\beta 3 .{ }^{13}$ On the other hand, both $\beta 1$ and $\beta 5$ integrins occur in osteoclasts and may have important functions when coupled with $\alpha \mathrm{v}$ or other $\alpha$ family integrins that are expressed at lower, but still significant, levels in osteoclasts. ${ }^{24,25}$ There were increases in $\beta 1$ and $\beta 5$ expression in this osteopetrosis with attachment defects, but an abnormally processed form of $\beta 3$ was also a major $\beta$ chain (Figure 6). However, podosomes did not react with an $\alpha \mathrm{v} \beta 3$ monoclonal antibody that labels normal osteoclast podosomes reliably (Figure 4). This probably reflected abnormal processing of $\beta 3$, as $\beta 3$ was mostly smaller than normal (Figure 6). However, the $\beta 3$ sequence was normal in all exons, so we considered an integrin assembly or processing defect. Owing to post-translational processing, integrins in different cell types have different sizes and structures (compare HeLa and MG63 controls to osteoclast lysates in Figure 6). Increased quantities of $\beta 1$ and $\beta 5$ integrin chains also occur with $\beta 3$ defects, although there was no major change in $\alpha 2$ integrin expression, which is reported in Glantzmann's thrombasthenia, ${ }^{13}$ and the patient had, apparently, normal clotting function. Thus, the observations were inconsistent with an isolated attachment protein defect.

The key finding, however, in this new type of osteopetrosis was that organization of podosomes lacked the annular structure and association with actin rings that are needed for normal osteoclast function (Figure 4). To provide additional insights into a possible molecular defect underlying these findings, we considered that integrin assembly and function in osteoclasts reflects a complex developmental and regulatory system, which is needed for assembly of the annular integrin ring and for the system to be disassembled for motility. Several proteins contribute to the regulation and assembly of the integrin complex. Key elements include the non-receptor tyrosine kinase Src, the rho family guanine exchange factor Vav3, and the adaptor protein Vasp. ${ }^{9,11,26,27}$ Vasp is regulated by the cGMP-dependent protein kinase and is needed for motility. ${ }^{27,28}$ As the cells in this case made pits, albeit small ones, and were motile (Figures 1 and 2), defects in the proteins $\mathrm{Src}^{29}$ and Vasp appeared less likely to contribute to the phenotype. However, Vav3 regulates cell spreading and integrin assembly. ${ }^{26}$ Study of Vav3 in the osteopetrotic cells showed that Vav3 distribution was dysfunctional. Association with peripheral podosomes and actin organizing structures was totally lost (Figure 5), possibly reflecting an isoform of Vav3 $\sim 20 \mathrm{kD}$ smaller than the normal protein (Figure 6). However, as with $\beta 3$, this was not because of defective primary sequence in the exons of the genomic DNA. av3-deficient mice have increased bone mass; however, not frank osteopetrosis, ${ }^{11}$ although the phenotype is not known in humans. The increased bone mass in Vav3-deficient mice might also reflect possible anabolic effects on bone resorption of non-resorbing osteoclasts. ${ }^{30}$ The additional proteins associated with osteoclast organizational defects, Plekhm $1^{8}$ and $\mathrm{Src},{ }^{29}$ were also sequenced and were normal.

Possible reasons for this phenotype include abnormal regulation of the attachment-detachment cycle, which includes limited proteolytic activity, or an abnormality in an unknown organizing protein upstream of the attachment complex. Proteolytic activity during cell detachment clearly occurs ${ }^{28}$ although normally any protein cleaved should be rapidly cleared and replaced. We cannot exclude the presence of a mutation in introns of $\beta 3$ or Vav3, although it is unlikely. We also cannot exclude a mutation in a regulatory region (promoter, enhancer etc), although this is even less likely, as the quantities of proteins produced were normal. In any case, the important membrane integrin assembly complex must involve further elements, and other adaptor-protein interactions that cause osteoclastic dysfunction, which remains to be described. Although commonly related to absence of the $\beta 3$ integrin, clotting disorders with osteopetrosis are also described with $\alpha 2$ integrins and with $\beta 3$ defects other than deletions. ${ }^{31}$ Other candidate integrins regulating osteoclasts include $\alpha 9 \beta 1 .^{32}$ How these, in addition to $\alpha 2$, may affect an osteoclast with an $\alpha \mathrm{v} \beta 3$ expression defect, are likely related to downstream intermediate proteins and kinases, which may vary specifically as integrin assembly specific molecules. Integrin-related pathways beyond Src, Vav3, and Vasp include the integrin-linked kinase $^{32}$ and disintegrins including Adam8. ${ }^{33}$ Adam8 is linked to pathological resorption than to deficient resorption. ${ }^{33,34}$ Surface distribution of integrins is also regulated to some extent by the integrin linked kinase, ${ }^{35}$ and it is expressed in the osteoclast, although its role in modulating adhesion or activity is not known. In macrophages, it is modulated by NF- $\kappa \mathrm{B}$ and linked to NO production. ${ }^{36}$

It is also entirely possible, as we sequenced the major genes known to cause related defects directly, that none of the attachment protein complex elements are abnormal. This could also be an epigenomic abnormality, such as microRNA suppression of gene expression that may mimic a genetic defect. This is presently known in osteoclasts only as a laboratory-created effect. ${ }^{37}$ On the other hand, such effects are described with changes in expression because of organspecific anomalies in micro-RNA expression that affect specific pathways, including integrin expression and cell attachment, in some cells. ${ }^{38,39}$ Such changes in attachment proteins have been related to cell-transformation effects, but there is no reason to believe that similar effects might not affect the activity of cells that depend on rapid turnover and replacement of attachment proteins for motility, such as osteoclasts, although this possible mechanism is at present entirely hypothetical. 
It is important to note that overall assembly of attachment is regulated by the Vav family of guanine nucleotide exchange factors. ${ }^{40-42}$ Although cell-specific defects of integrin assembly related to these elements are not previously described to cause human disease, Vav3 deficient mice have abnormal polarization, increased bone mass, and decreased pit size from osteoclasts made in vitro. ${ }^{11}$ The novel type of osteopetrosis described here had abnormal integrin distribution and microscopic resorption pits similar to osteoclasts from the Vav3-deficient mouse. The affected osteoclasts had no peripheral rings of podosomes, and synthesized normal quantities of $\alpha \mathrm{v}$ and $\beta 3$, but the $\beta 3$ was processed abnormally. This reflected an abnormal distribution of Vav3, with an abnormal Vav3 isoform at $\sim 70 \mathrm{kD}$. These findings suggest that truncated Vav3 may be responsible, at least in part, for this new type of human osteopetrosis. However, as the exon sequence of Vav3 was normal, this is either due to an undetected intron defect, or it results from accumulation of partially degraded Vav3 protein. In support of this possibility, a short form of the $\beta 3$ integrin also accumulated. We conclude that, in this new type of osteopetrosis, the integrinorganizing complex is dysfunctional, and at least two proteins within the complex included truncated, possibly retained partially degraded, proteins.

\section{ACKNOWLEDGEMENT}

Supported in part by a NOBEL grant from Fondazione Cariplo, and by the Department of Veteran's Affairs (USA) and the National Institutes of Health (USA) AR053976, AR055208, and AR053566.

1. Tolar J, Teitelbaum SL, Orchard PJ. Osteopetrosis. N Engl J Med 2004;351:2839-2849.

2. Blair HC, Teitelbaum SL, Ghiselli $R$, et al. Osteoclastic bone resorption by a polarized vacuolar proton pump. Science 1989;245:855-857.

3. Li YP, Chen W, Liang $Y$, et al. Atp6i-deficient mice exhibit severe osteopetrosis due to loss of osteoclast-mediated extracellular acidification. Nat Genet 1999;23:447-451.

4. Blair HC, Borysenko CW, Villa $\mathrm{A}$, et al. In vitro differentiation of CD14 cells from osteopetrotic subjects: contrasting phenotypes with TCIRG1, CLCN7, and attachment defects. J Bone Miner Res 2004;19:1329-1338.

5. Scheel O, Zdebik AA, Lourdel S, et al. Voltage-dependent electrogenic chloride/proton exchange by endosomal CLC proteins. Nature 2005;436:424-427.

6. Kornak U, Kasper D, Bosl MR, et al. Loss of the CIC-7 chloride channel leads to osteopetrosis in mice and man. Cell 2001;104:205-215.

7. Lange PF, Wartosch L, Jentsch TJ, et al. CIC-7 requires Ostm1 as a betasubunit to support bone resorption and lysosomal function. Nature 2006;440:220-223.

8. Van Wesenbeeck L, Odgren PR, Coxon FP, et al. Involvement of PLEKHM1 in osteoclastic vesicular transport and osteopetrosis in incisors absent rats and humans. J Clin Invest 2007;117:919-930.

9. Blair HC, Zaidi M. Osteoclastic differentiation and function regulated by old and new pathways. Rev Endocr Metab Disord 2006;7:23-32

10. Sobacchi C, Frattini A, Guerrini MM, et al. Osteoclast-poor human osteopetrosis due to mutations in the gene encoding RANKL. Nat Genet 2007;39:960-962.

11. Faccio $R$, Teitelbaum SL, Fujikawa $K$, et al. Vav3 regulates osteoclast function and bone mass. Nat Med 2005;11:284-290.

12. Guerrini MM, Sobacchi C, Cassani B, et al. Human osteoclast-poor osteopetrosis with hypogammaglobulinemia due to TNFRSF11A (RANK) mutations. Am J Hum Genet 2008;83:64-76.
13. Horton MA, Massey HM, Rosenberg $\mathrm{N}$, et al. Upregulation of osteoclast $\alpha 2 \beta 1$ integrin compensates for lack of $\alpha v \beta 3$ vitronectin receptor in Iraqi-Jewish-type Glanzmann thrombasthenia. Br J Haematol 2003;122:950-957.

14. Frattini A, Orchard PJ, Sobacchi C, et al. Defects in TCIRG1 subunit of the vacuolar proton pump are responsible for a subset of human autosomal recessive osteopetrosis. Nat Genet 2000;25:343-346.

15. Robinson $\sqcup$, Yaroslavskiy BB, Griswold RD, et al. Estrogen inhibits RANKL-stimulated osteoclastic differentiation of human monocytes through estrogen and RANKL-regulated interaction of ER- $\alpha$ with BCAR1 and Traf6. Exp Cell Res 2009;315:1287-1301.

16. Yaroslavskiy BB, Li Y, Ferguson DJP, et al. Autocrine and paracrine nitric oxide regulate attachment of human osteoclasts. J Cell Biochem 2004;91:962-972.

17. Carano A, Schlesinger PH, Athanasou NA, et al. Acid and base effects on avian osteoclast activity. Am J Physiol 1993;264:C694-C701.

18. Kudo O, Fujikawa $Y$, Itonaga I, et al. Proinflammatory cytokine (TNF $\alpha /$ IL-1a) induction of human osteoclast formation. J Pathol 2002;198:220-227.

19. Frattini $A$, Vezzoni $P$, Villa $A$, et al. The dissection of human autosomal recessive osteopetrosis identifies an osteoclast-poor form due to RANKL deficiency. Cell Cycle 2007;6:3027-3033.

20. Blair HC, Athanasou NA. Recent advances in osteoclast biology and pathological bone resorption. Histol Histopathol 2004;19: 189-199.

21. Ross FP, Chappel J, Alvarez Jl, et al. Interactions between the bone matrix proteins osteopontin and bone sialoprotein and the osteoclast integrin $\alpha \mathrm{v} \beta 3$ potentiate bone resorption. J Biol Chem 1993:268:9901-9907.

22. Roux S, Pichaud F, Quinn J, et al. Effects of prostaglandins on human hematopoietic osteoclast precursors. Endocrinology 1997;138:1476-1482.

23. Bader BL, Rayburn H, Crowley D, et al. Extensive vasculogenesis, angiogenesis, and organogenesis precede lethality in mice lacking all $\alpha v$ integrins. Cell 1998;95:507-519.

24. Nakayamada S, Okada $\mathrm{Y}$, Saito $\mathrm{K}$, et al. $\beta 1$ integrin/focal adhesion kinase-mediated signaling induces intercellular adhesion molecule 1 and receptor activator of nuclear factor $\kappa \mathrm{B}$ ligand on osteoblasts and osteoclast maturation. J Biol Chem 2003;278:45368-45374.

25. Sago K, Teitelbaum SL, Venstrom K, et al. The integrin $\alpha v \beta 5$ is expressed on avian osteoclast precursors and regulated by retinoic acid. J Bone Miner Res 1999;14:32-38.

26. Felsenfeld DP, Schwartzberg PL, Venegas A, et al. Selective regulation of integrin-cytoskeleton interactions by the tyrosine kinase Src. Nat Cell Biol 1999;1:200-206.

27. Yaroslavskiy BB, Zhang Y, Kalla SE, et al. NO-dependent osteoclast motility: reliance on CGMP-dependent protein kinase I and VASP. J Cell Sci 2005;118:5479-5487.

28. Yaroslavskiy BB, Sharrow AC, Wells A, et al. Necessity of inositol $(1,4,5)$ trisphosphate receptor 1 and mu-calpain in NO-induced osteoclast motility. J Cell Sci 2007;120:2884-2894.

29. Miyazaki T, Tanaka S, Sanjay A, et al. The role of c-Src kinase in the regulation of osteoclast function. Mod Rheumatol 2006;16 :68-74.

30. Karsdal MA, Martin TJ, Bollerslev J, et al. Are nonresorbing osteoclasts sources of bone anabolic activity? J Bone Miner Res 2007;22: 487-494.

31. Yarali N, Fisqin T, Duru F, et al. Osteopetrosis and Glanzmann's thrombasthenia in a child. Ann Hematol 2003;82:254-256.

32. Boulter $E$, Van Obberghen-Schilling E. Integrin-linked kinase and its partners: a modular platform regulating cell-matrix adhesion dynamics and cytoskeletal organization. Eur J Cell Biol 2006;85:255-263.

33. Mandelin J, Li TF, Hukkanen MV, et al. Increased expression of a novel osteoclast-stimulating factor, ADAM8, in interface tissue around loosened hip prostheses. J Rheumatol 2003;30: 2033-2038.

34. Ainola M, Li TF, Mandelin J, et al. Involvement of ADAM8 in osteoclastogenesis and pathological bone destruction. Ann Rheum Dis 2009;68:427-434.

35. Vouret-Craviari V, Boulter E, Grall D, et al. ILK is required for the assembly of matrix-forming adhesions and capillary morphogenesis in endothelial cells. J Cell Sci 2004;117:559-569. 
36. Tan C, Mui A, Dedhar S. Integrin-linked kinase regulates inducible nitric oxide synthase and cyclooxygenase-2 expression in an NF- $\kappa \mathrm{B}$ dependent manner. J Biol Chem 2002;277:3109-3116.

37. Sugatani T, Hruska KA. Impaired micro-RNA pathways diminish osteoclast differentiation and function. J Biol Chem 2009;284:4667-4678.

38. Müller DW, Bosserhoff AK. Integrin beta 3 expression is regulated by let-7a miRNA in malignant melanoma. Oncogene 2008;27:6698-6706.

39. Brendle $A$, Lei $H$, Brandt $A$, et al. Polymorphisms in predicted microRNA-binding sites in integrin genes and breast cancer. Carcinogenesis 2008;29:1394-1399.
40. Miranti CK, Leng $\mathrm{L}$, Maschberger $\mathrm{P}$, et al. Identification of a novel integrin signaling pathway involving the kinase Syk and the guanine nucleotide exchange factor Vav1. Curr Biol 1998;8: 1289-1299.

41. Ardouin $L$, Bracke $M$, Mathiot $A$, et al. Vav1 transduces TCR signals required for LFA-1 function and cell polarization at the immunological synapse. Eur J Immunol 2003;33:790-797.

42. Graham DB, Robertson CM, Bautista J, et al. Neutrophil-mediated oxidative burst and host defense are controlled by a Vav-PLCgamma2 signaling axis in mice. J Clin Invest 2007;117:3445-3452. 